\title{
The importance of the toroidal magnetic field for the feasibility of a tokamak burning plasma experiment
}

\author{
E. Mazzucato \\ Princeton Plasma Physics Laboratory, Princeton University \\ P. O. Box 451, Princeton, New Jersey 08543
}

\begin{abstract}
The next step in the demonstration of the scientific feasibility of a tokamak fusion reactor is a DT burning plasma experiment for the study and control of self-heated plasmas. In this paper, we examine the role of the toroidal magnetic field on the confinement of a tokamak plasma in the ELMy H-mode regime - the operational regime foreseen for ITER.
\end{abstract}

It is commonly taken for granted that the next step in the development of a tokamak fusion reactor is a DT burning plasma experiment, where charged fusion products (i.e., $\alpha$ particles) are the main source of plasma heating. Several of these experiments have been proposed over the years - ITER being the most prominent [1].

The operational mode foreseen for ITER is the ELMy H-mode, for which a number of empirical scaling laws have been published. In general, such scaling laws are cast in the form [2]

$$
\tau_{E} \omega_{c}=\rho^{*-\alpha} F\left(\beta, v^{*},\left\{p_{i}\right\}\right),
$$

where $\tau_{E}$ is the plasma energy confinement time, $\omega_{c}$ is the ion cyclotron frequency, $\rho^{*}=\rho / a$ is the normalized ion Larmor radius ( $a=$ minor radius), and $F$ is a function of the toroidal plasma beta $\beta$, the effective collision frequency $v^{*}$, and a set $\left\{p_{i}\right\}$ of dimensionless parameters including the safety factor $q_{95}$, the aspect ratio $A$, the elongation $k$, the triangularity $\delta$ and the average isotopic number $M$. In the following, we will use the scaling given in Ref. [2]

$$
\tau_{E} \omega_{c} \propto \rho^{*-2.70} \beta^{-0.90} v^{*-0.01} M^{0.96} q_{95}^{-3.0} A^{-0.73} k^{2.3}
$$

where for simplicity we will neglect the weak dependence on $v^{*}$. 
In the operation of tokamaks, one parameter of crucial importance is the ratio of the line average plasma density $(\bar{n})$ to the Greenwald limit $n_{G W}=I_{p} / \pi a^{2}\left[10^{20} \mathrm{~m}^{-3}\right.$, MA, $\mathrm{m}^{-2}$ ] (where $I_{p}$ is the toroidal plasma current). Experiments [3] indicate that the plasma confinement degrades very quickly as this ratio approaches unity. In the ITER-EDA design [1], the value of $n_{G}=\bar{n} / n_{G W}$ was chosen unrealistically larger than one. In later versions of ITER (IAM [4], LAM [4] and FEAT [5]), this ratio was lowered to 0.85 - a value that unfortunately is still too large. Another serious problem in ITER is the need for large normalized betas $\left(\beta_{N}=\beta /\left(I_{p} / B a\right)\right)$, with values that are at the upper extreme of the database. Finally, the third problem is the large size and high cost of the apparatus, which is what has prevented the construction of ITER so far. All three of these problems - large density, beta and size - could lessen by operating at larger toroidal magnetic fields $(B)$.

To see how important is the toroidal magnetic field for the scientific feasibility of a burning plasma experiment, let us consider the energy balance of a DT tokamak and assume that the plasma thermal losses, represented by Eq. (2), are the dominant loss of plasma energy. We have

$$
B^{2} \beta \tau_{E} \propto G / N
$$

where $G=Q /(5+Q), Q$ is the energy gain, $N=<n^{2} \overline{\sigma v}>/ B^{4} \beta^{2}$, $\overline{\sigma V}$ is the nuclear fusion rate and the brackets $<>$ indicate the volume average.

One important engineering parameter is the average neutron wall loading $P_{w}$, which in ITER-EDA [1] is $1.0 \mathrm{MW} / \mathrm{m}^{2}$, and $0.5 \mathrm{MW} / \mathrm{m}^{2}$ in later versions of this project $[4,5]$. At constant values of $q_{95}, A$ and $k$, the neutron wall loading scales as

$$
P_{w} \propto N B^{4} \beta^{2} a .
$$

This, together with Eq. (2) and (3), gives 


$$
\begin{aligned}
a \propto \frac{T^{0.51}(G / N)^{0.38}}{B^{1.32}\left(P_{w} / N\right)^{0.02}} \\
\beta_{N} \propto \frac{\left(P_{w} / N\right)^{0.51}}{B^{1.34} T^{0.25}(G / N)^{0.19}} . \\
n_{G} \propto \frac{\left(P_{w} / N\right)^{0.49}(G / N)^{0.19}}{B^{1.66} T^{0.74}}
\end{aligned}
$$

From these equations, where the temperature $T$ is a free parameter that we assume varying in a restricted range where $N$ is constant, we see that the plasma linear dimension $(a)$, and the normalized values of plasma beta $\left(\beta_{N}\right)$ and density $\left(n_{G}\right)$ are all decreasing functions of the magnetic field. On the contrary, $a$ increases with $T$, while the opposite is true for $\beta_{N}$ and $n_{G}$. Finally, Eqs. (5) show that the plasma linear dimension is insensitive to the neutron wall loading

Tables 1 and 2 show the result of scaling according to Eqs. (5) the reference discharges of ITER-RC-IAM [4] and FEAT [5] to three tokamaks with a toroidal magnetic field of $13 \mathrm{~T}$ (as in Ignitor [6]), $10 \mathrm{~T}$ (as in FIRE [7]) and $8 \mathrm{~T}$. The scaling is performed keeping the values of $A, k, q_{95}$ and $Q(=10)$ constant. Other quantities in the tables are the average plasma temperature $T_{n} \equiv<n T>/<n>$, the poloidal plasma beta $\beta_{p}$, the vacuum magnetic energy $E_{T F}$, the fusion power $P_{f}$, and the $\mathrm{L}-\mathrm{H}$ transition power $P_{L H}$ for which we use the expression $P_{L H}=3.24 M^{-1} B^{0.75} \bar{n}_{20}^{0.60} R^{0.98} a^{0.81}$ given in Ref. [2]. We consider both $T_{n}$ and $P_{w}$ as input parameters, and for the latter we impose the constraint of making the total plasma heating power (i.e., the sum of the power of $\alpha$-particles and that of auxiliary heating) larger than $P_{L H}$.

The linear dimensions of the case with $B=13 \mathrm{~T}$ are very similar to those of Ignitor. This is indeed an extraordinary result since the size of the latter has been determined using a completely different transport model [6]. Consequently, considering that Ignitor 
has a larger plasma current (11 MA) as well, we conclude that this experiment has the potential for reaching the same goals of ITER-RC and FEAT, i.e., $Q=10$. However, this is contingent upon accessing the H-mode ELMy regime, a possibility that might be jeopardized by the absence of a divertor in the Ignitor design.

On the contrary, both the linear dimensions and the plasma current of the case with $B=10 \mathrm{~T}$ (fourth column) are substantially larger than those of FIRE [7]. Consequently, for the latter we reach the opposite conclusion than for Ignitor, i.e., that the scaling law of Eq. (2) will prevent FIRE from reaching $Q=10$.

Finally, the third column in the tables refers to a tokamak with characteristics that are intermediate between those of ITER and Ignitor. Compared to the former, it needs lower values of $\beta_{N}$ and $n_{G}$, and has a much smaller size (by almost a factor of two). Compared to Ignitor, its TF coils are under smaller mechanical stresses (more than a factor of 2.5), and the dissipated Ohmic power is much weaker (by more than an order of magnitude). This should allow a pulse flattop duration of at least $50 \mathrm{~s}$ under adiabatic conditions.

Throughout Tables 1 and 2 we have used $q_{95}=3.0$, which is the value used in all versions of ITER. As pointed out several times by the proponents of Ignitor [6], such a low safety factor could result in a large region with $q<1$, where the confinement of $\alpha$ particles could be jeopardized by the deleterious effects of internal $m=1$ plasma instabilities. However, the safety factor can be increased by lowering the aspect ratio and/or increasing the elongation. For instance, in Table 2 we get $q_{95}=3.6$ (the value used in Ignitor [6]) with a 5\% enlargement of the minor radius and by increasing the elongation to 1.8. Fortunately, both changes are beneficial for the energy confinement time (Eq. (2)).

In conclusion, the large values of $n_{G}$ and $\beta_{N}$ that are necessary for obtaining an energy gain of 10 in ITER-RC and FEAT cast serious doubts on the possibility of 
reaching the programmatic objectives of these experiments. This is a direct consequence of the confinement scaling laws that have been chosen as the physics basis of these experiments and the low value of toroidal magnetic field. The same scaling laws predict that the plasma linear dimension and the normalized values of plasma density and beta are all decreasing functions of $B$. Hence, by using a larger toroidal magnetic field, one can operate in a safer plasma regime with a smaller device. Tables 1 and 2 contain three options varying from an aggressive Ignitor-like tokamak with $B=13 \mathrm{~T}$, to a less technically demanding device with $B=8 \mathrm{~T}$. The improved operating regime and the smaller plasma size should greatly enhance both the physical and practical feasibility of these experiments. Needless to say, superconducting magnets are difficult to use in any tokamak with a toroidal magnetic field of $8 \mathrm{~T}$, and certainly they become unfeasible at larger values of magnetic field. Nevertheless, since the primary objective of these experiments is the study of plasma transport in an $\alpha$-dominated regime, the most important requirement for their pulse duration is to be much longer than the plasma energy confinement time. Fortunately, for the proposed tokamaks in the present note this condition can be satisfied using cryogenically cooled normal conductors.

Finally, the last exercise in this paper is the scaling of the reference discharge of ITER-FEAT to three ignited tokamaks with the same values of magnetic field used previously. By constraining the power of $\alpha$-particles to be $20 \%$ larger than $P_{L H}$, we obtain the parameters of Table 3 showing similar values of $\beta_{N}, \beta_{p}$ and $n_{G}$ for all three tokamaks. Consequently, the preferred option must be selected on the basis of engineering and economic considerations.

The author would like to acknowledge useful conversations with B. Coppi, D. Meade, E. Salpietro and J. Schmidt. This work was supported by U.S. DOE Contract No. DEAC02-76-CHO-3073 


\section{References}

[1] Final Design Report, ITER EDA Documentation Series, IAEA, Vienna (1998).

[2] ITER Physics Expert Groups on Confinement and Transport, Nucl. Fusion 392175 (1999).

[3] G. Saibene, et al, Nucl. Fusion 39, 1133 (1999).

[4] Study of Options for the Reduced Technical Objectives/Reduced Cost (RTO/RC) ITER, Report by the Director, ITER Council Meeting No. 15 (1999).

[5] ITER-FEAT Outline Design Report, ITER Meeting, Tokyo, (January 2000).

[6] B. Coppi, B. Nassi and L. E. Sugiyama, Phys. Scr. 45, 112 (1992).

[7] D. Meade and R Woolley, Proceedings of the $20^{\text {th }}$ Symposium on Fusion Technology, Marseilles, France (1998). 
TABLE 1. ELMY H-mode scaling of the ITER-RC-IAM reference discharge to three tokamaks with a toroidal magnetic field $(B)$ of 8,10 and $13 \mathrm{~T}$, respectively; values of aspect ratio $(A)$, elongation $(k)$, triangularity, safety factor $\left(q_{95}\right)$ and energy gain $(Q)$ are kept constant.

\begin{tabular}{|c|c|c|c|c|}
\hline & $\begin{array}{c}\text { ITER } \\
\text { IAM }\end{array}$ & $\begin{array}{c}\text { MEDIUM } \\
B \\
\end{array}$ & $\begin{array}{c}\text { HIGH } \\
B \\
\end{array}$ & $\begin{array}{c}\text { VERY } \\
\text { HIGH } B\end{array}$ \\
\hline $\begin{array}{ll}B & {[\mathrm{~T}]}\end{array}$ & 5.51 & 8.00 & 10.0 & 13.0 \\
\hline$T_{n} \quad[\mathrm{keV}]$ & 10.0 & 8.0 & 8.0 & 7.0 \\
\hline$a \quad[\mathrm{~m}]$ & 1.90 & 1.04 & 0.77 & 0.50 \\
\hline$R \quad[\mathrm{~m}]$ & 6.20 & 3.38 & 2.50 & 1.63 \\
\hline$A$ & 3.26 & 3.26 & 3.26 & 3.26 \\
\hline$k$ & 1.83 & 1.83 & 1.83 & 1.83 \\
\hline$\beta \quad[\%]$ & 2.86 & 1.84 & 1.67 & 1.73 \\
\hline$\beta_{p}$ & 0.95 & 0.61 & 0.55 & 0.57 \\
\hline$\beta_{N}$ & 2.25 & 1.44 & 1.31 & 1.36 \\
\hline$n_{G}$ & 0.87 & 0.55 & 0.46 & 0.46 \\
\hline$q_{95}$ & 3.0 & 3.0 & 3.0 & 3.0 \\
\hline$I_{p} \quad[\mathrm{MA}]$ & 13.3 & 10.5 & 9.7 & 8.2 \\
\hline$E_{T F}[\mathrm{GJ}]$ & 44 & 15.0 & 9.5 & 4.5 \\
\hline$Q$ & 10 & 10 & 10 & 10 \\
\hline$P_{w} \quad\left[\mathrm{MW} / \mathrm{m}^{2}\right]$ & 0.50 & 0.50 & 0.75 & 1.50 \\
\hline$P_{f} \quad[\mathrm{MW}]$ & 500 & 148 & 122 & 104 \\
\hline$P_{L H}[\mathrm{MW}]$ & 48 & 29 & 25 & 21 \\
\hline
\end{tabular}


TABLE 2. ELMY H-mode scaling of the ITER-FEAT reference discharge to three tokamaks with a toroidal magnetic field $(B)$ of 8,10 and $13 \mathrm{~T}$, respectively; values of aspect ratio $(A)$, elongation $(k)$, triangularity, safety factor $\left(q_{95}\right)$ and energy gain $(Q)$ are kept constant.

\begin{tabular}{|c|c|c|c|c|c|}
\hline & & $\begin{array}{l}\text { ITER } \\
\text { FEAT } \\
\end{array}$ & $\begin{array}{c}\text { MEDIUM } \\
B \\
\end{array}$ & $\begin{array}{c}\text { HIGH } \\
B \\
\end{array}$ & $\begin{array}{c}\text { VERY } \\
\text { HIGH } B\end{array}$ \\
\hline$B$ & {$[\mathrm{~T}]$} & 5.30 & 8.00 & 10.0 & 13.0 \\
\hline$T_{n}$ & {$[\mathrm{keV}]$} & 9.0 & 8.0 & 8.0 & 7.0 \\
\hline$a$ & {$[\mathrm{~m}]$} & 2.00 & 1.09 & 0.81 & 0.53 \\
\hline$R$ & {$[\mathrm{~m}]$} & 6.20 & 3.39 & 2.51 & 1.63 \\
\hline$A$ & & 3.10 & 3.10 & 3.10 & 3.10 \\
\hline$k$ & & 1.70 & 1.70 & 1.70 & 1.70 \\
\hline$\beta$ & {$[\%]$} & 2.50 & 1.48 & 1.35 & 1.40 \\
\hline$\beta_{p}$ & & 0.67 & 0.40 & 0.36 & 0.37 \\
\hline$\beta_{N}$ & & 1.77 & 1.05 & 0.96 & 0.99 \\
\hline$n_{G}$ & & 0.85 & 0.47 & 0.39 & 0.39 \\
\hline$q_{95}$ & & 3.0 & 3.0 & 3.0 & 3.0 \\
\hline & [MA] & 15.0 & 12.4 & 11.5 & 9.7 \\
\hline$E_{T F}$ & {$[\mathrm{GJ}]$} & 40 & 15.0 & 9.4 & 4.4 \\
\hline$Q$ & & 10 & 10 & 10 & 10 \\
\hline$P_{w}$ & {$\left[\mathrm{MW} / \mathrm{m}^{2}\right]$} & 0.50 & 0.50 & 0.75 & 1.50 \\
\hline$P_{f}$ & [MW] & 410 & 122 & 100 & 85 \\
\hline$P_{L H}$ & [MW] & 48 & 28 & 24 & 21 \\
\hline
\end{tabular}


TABLE 3. ELMY H-mode scaling of the ITER-FEAT reference discharge to three ignited tokamaks with a toroidal magnetic field $(B)$ of 8,10 and $13 \mathrm{~T}$, respectively; values of aspect ratio $(A)$, elongation $(k)$, triangularity, and safety factor $\left(q_{95}\right)$ are kept constant.

\begin{tabular}{lllll}
\hline & & $\begin{array}{c}\text { TOK } \\
\# 1\end{array}$ & $\begin{array}{c}\text { TOK } \\
\# 2\end{array}$ & $\begin{array}{l}\text { TOK } \\
\# 3\end{array}$ \\
\hline$B$ & {$[\mathrm{~T}]$} & 8.00 & 10.0 & 13.0 \\
$T_{n}$ & {$[\mathrm{keV}]$} & 8.0 & 8.0 & 8.0 \\
$a$ & {$[\mathrm{~m}]$} & 1.26 & 0.93 & 0.65 \\
$R$ & {$[\mathrm{~m}]$} & 3.92 & 2.89 & 2.02 \\
$A$ & & 3.10 & 3.10 & 3.10 \\
$k$ & & 1.70 & 1.70 & 1.70 \\
$\beta$ & {$[\%]$} & 1.69 & 1.62 & 1.45 \\
$\beta_{p}$ & & 0.45 & 0.44 & 0.39 \\
$\beta_{N}$ & & 1.20 & 1.15 & 1.03 \\
$n_{G}$ & & 0.62 & 0.55 & 0.44 \\
$q_{95}$ & & 3.0 & 3.0 & 3.0 \\
$I_{p}$ & {$[\mathrm{MA}]$} & 14.3 & 13.2 & 12.0 \\
$E_{T F}$ & {$[\mathrm{GJ}]$} & 23.0 & 14.5 & 8.4 \\
$Q$ & & $\infty$ & $\infty$ & $\infty$ \\
$P_{w}$ & {$\left[\mathrm{MW} / \mathrm{m}^{2}\right]$} & 0.750 & 1.25 & 2.00 \\
$P_{f}$ & {$[\mathrm{MW}]$} & 246 & 223 & 175 \\
$P_{L H}$ & {$[\mathrm{MW}]$} & 40 & 35 & 29 \\
\hline
\end{tabular}

